\title{
LA TEORÍA DEL CAPITAL CULTURAL Y LA PARTICIPACIÓN CULTURAL DE LOS JÓVENES. EL CASO CHILENO COMO EJEMPLO
}

MODESTO GAYO*

\begin{abstract}
RESUMEN
La teoría del capital cultural desarrollada por Pierre Bourdieu y sus seguidores ha mantenido una relación ambivalente con la juventud. Por un lado, las etapas iniciales de la vida son cruciales para la conformación del habitus y, con ello, sientan las bases para los logros futuros. Por otro lado, los jóvenes ocupan posiciones subordinadas en el campo social y son recipientes de una cultura heredada desde sus familias y contextos sociales de origen. Sin embargo, los estudios realizados en los últimos años en el marco de esta teoría, principalmente los que se refieren a la práctica cultural, han mostrado la importancia de la edad para la comprensión de los patrones de comportamiento identificados. A pesar de ello, históricamente han sido las investigaciones hechas desde la teoría de las subculturas las que más han avanzado en el estudio de los jóvenes. Este artículo intenta contribuir al desarrollo de la teoría del capital cultural recurriendo mayormente al diálogo y la convergencia metodológica entre ambas teorías, y lo hace con evidencia del caso chileno.

\footnotetext{
PALABRAS CLAVE: JUVENTUD, CAPITAL CULTURAL, PARTICIPACIÓN CULTURAL, ESPACIO SOCIAL, CAMPO SOCIAL
}

* Modesto Gayo es profesor en la Escuela de Sociología de la Universidad Diego Portales, Santiago, Chile. E-Mail: modesto.gayo@udp.cl.

Este estudio se hizo en el marco del proyecto Fondecyt $\mathrm{N}^{\mathrm{o}} 1100523$, titulado «La desigualdad cultural y sus determinantes. Un estudio sobre el gusto y la participación cultural en Chile». Asimismo, quiero agradecer a Camila Malig su ayuda en la revisión de un amplio conjunto de textos que están en la base de este artículo, y a Sahara Martignoni por su trabajo en la preparación de la base de datos, ambas jóvenes investigadoras.
\end{abstract}




\title{
A TEORIA DO CAPITAL CULTURAL E A PARTICIPAÇÃO CULTURAL DOS JOVENS. O CASO CHILENO COMO EXEMPLO
}

\begin{abstract}
RESUMO
A teoria do capital cultural - desenvolvida por Pierre Bourdieu e seus seguidores - , tem mantido uma relação ambivalente com a juventude. Por um lado, os estágios iniciais de vida são cruciais para a formação do habitus, que estabelece as bases para futuras realizações. Por outro lado, os jovens ocupam posições subalternas no campo social, sendo herdeiros de uma cultura transmitida por suas famílias e contextos sociais de origem. No entanto, os estudos realizados nos últimos anos no contexto dessa teoria - principalmente em relação à prática cultural—, têm mostrado a importância da idade para compreensão dos padrões de comportamento identificados. Apesar disso, historicamente, as pesquisas feitas a partir da teoria das subculturas são as que mais têm avançado no estudo dos jovens. O objetivo deste artigo é contribuir para o desenvolvimento da teoria do capital cultural recorrendo majoritariamente ao diálogo e à convergência metodológica entre ambas as teorias, usando como exemplo o caso chileno.

\section{PALAVRAS CHAVE: JUVENTUDE, CAPITAL CULTURAL, PARTICIPAÇÃO CULTURAL, ESPAÇO SOCIAL, CAMPO SOCIAL \\ THE THEORY OF CULTURAL CAPITAL \\ AND THE CULTURAL PARTICIPATION OF YOUNG PEOPLE. THE CHILEAN CASE AS AN EXAMPLE}

\begin{abstract}
Bourdieu's cultural capital theory has had an ambivalent relationship with the idea of «youth». On the one hand, the first steps in life are key moments for habitus building and, doing that, set the basis for future achievements. On the other hand, youngsters occupy subordinated positions in the social field and they are recipients of particular cultures from their families and social contexts of breeding. However, a significant number of studies on cultural capital performed during the last few years have concluded that age is a very important variable in order to account for the patterns of behavior found. Even so, in the past, studies done from the theory of subcultures have been those which have made more progress in understanding youth cultures. This article attempts to contribute to the development of cultural capital theory taking advantage from the dialogue and methodological convergence between both theories, and this is done using evidence from the Chilean case.
\end{abstract}

KEY WORDS: YOUTH, CULTURAL CAPITAL, CULTURAL PARTICIPATION, SOCIAL SPACE, SOCIAL FIELD 


\section{INTRODUCCIÓN}

LA TEORÍA DEL CAPITAL cultural a la que vamos a referirnos aquí es la que en la tradición sociológica habitualmente asociamos al pensador francés Pierre Bourdieu. De acuerdo a esta teoría, las prácticas culturales de las personas son un producto de $-\mathrm{o}$ se ven fuertemente influidas por- la interiorización inconsciente de esquemas cognitivos, valóricos y afectivos, que en su conjunto de lo que podemos entender como «disposiciones» y de las constricciones estructurales relativas principalmente a la dotación diferencial de los capitales cultural y económico (Bourdieu, 1979). Eso significa que las personas con mayor educación formal, más recursos económicos y un origen social más elevado tendrán un comportamiento cultural que podríamos entender como más sofisticado, refinado o, haciendo uso de un término polémico pero de gran tradición, legítimo.

El objetivo de este estudio no es poner a prueba esta teoría en los términos arriba indicados, trabajo que ya se ha hecho en otros lugares con gran detenimiento (Bennett et al., 2009; Gayo et al., 2009), sino que se trata de contribuir a desarrollar la teoría del capital cultural con referencia a los jóvenes.

Para ello, en primer lugar, mostramos el papel ambivalente y subordinado que la juventud como categoría de análisis ha tenido en este desarrollo teórico. En segundo lugar, argumentamos la insuficiencia de estudios previos para hacer énfasis en la juventud sin necesidad de desplazar del centro de interés el tratamiento de las desigualdades, quizás porque entendieron que estas eran transversales a los grupos etarios. No obstante, en tercer lugar, incluso si aceptamos la idea de transversalidad $\mathrm{u}$ ortogonalidad de las variables edad y grupo socioeconómico, esto ha tenido una consecuencia indeseable. Esta se refiere al hecho de que ha contribuido a mantener a los jóvenes en una especie de posición analítica que ha obstaculizado estudiar las diferencias socioeconómicas entre ellos, aunque es evidente que estas existen y tienen relevancia (Gayo et al., 2011; Winocur, en Sunkel 2006; Terpstra, 2006;), como aquí mostraremos. ${ }^{1}$ En cuarto lugar, presentamos los resultados de un estudio

1 No tratándose estricta o principalmente de desigualdades socioeconómicas, aunque pudiera haber relación con las mismas, existe literatura que trata sobre importantes diferencias entre los niños y/o los jóvenes. A este respecto, un ejemplo es el trabajo de Adriana Rodríguez (en Sunkel, 2006), en el cual trata la regulación que dentro de la familia se hace del 
empírico con datos de Chile recogidos recientemente y de los cuales se deriva una clara imagen de fuerte desigualdad entre los jóvenes (INJUV, 2010). Finalmente, extraemos algunas conclusiones, entre las cuales está la necesidad de reflexionar sobre la juventud no como una especie de limbo producido sociohistóricamente, ${ }^{2}$ y ajeno a la realidad de la estratificación social, sino como un momento en el desarrollo de los individuos en el cual las desigualdades reflejan su realidad cotidiana actual y nos ayudan a entender sus posibilidades de desempeño social y laboral en el futuro. Justamente, esto es de lo que la teoría del capital cultural habla, pero, quizás por tan evidente, ha llegado a ser olvidado u omitido. Este artículo trata de problematizar dicho sobreentendido.

\section{JUVENTUD Y LÍMITES DE LA TEORÍA DEL CAPITAL CULTURAL}

La teoría del capital cultural ha mostrado históricamente una relación ambivalente con la juventud (Bourdieu, 1979). Por un lado, la infancia y la juventud, o las primeras etapas de la socialización de los sujetos, son entendidas como momentos cruciales para explicar la formación del habitus; es decir, se trataría de una fase central en el desenvolvimiento de las disposiciones de las personas, sean estas estéticas, relativas a expectativas futuras, o concernientes a las prácticas que ordenan nuestra cotidianeidad. Sin embargo, por otro lado, el énfasis en la reproducción propio de esta teoría deja a la juventud como una fase personal en la que generalmente germina sobre todo lo que los padres asentaron. Esto último puede ser entendido como la «invisibilidad de la juventud», pues esta etapa carece de fuerza explicativa y funciona, más bien, como una correa de transmisión de algo cuya producción se debe a otras variables.

Los jóvenes son comprendidos como los novatos, los que entran en las disputas propias del campo cultural desde posiciones de relegamiento (Bourdieu, 2005). Ellos son los que carecen de una historia en el campo. Entran en el mismo y con ello se ven involucrados en una disputa por ganar legitimidad, y lo hacen desde abajo, mostrando frecuentemente pautas culturales que no encajan con un gusto generalmente aceptado hasta ese momento. Esto proponemos entenderlo

uso que los niños hacen de la televisión en Colombia, encontrándose fuertes diferencias en las reglas aplicadas para restringir o no el contacto con este medio de comunicación.

2 Para una reflexión sobre la historicidad de la «juventud» como categoría, puede verse Bourdieu (1990). 
como la «subordinación de la juventud» o la «juventud dominada», lo cual significa que el asentamiento y permanencia en el tiempo de los gustos nacientes deberá superar una etapa de rechazo por parte de las personas de edad superior, las cuales generalmente ocupan las posiciones dominantes y de más prestigio en el campo cultural.

Esta juventud invisible y subordinada poco parece tener que ver con la relevancia creciente que se ha venido otorgando a la edad en los estudios sobre capital cultural. La edad influye casi tanto como la clase en los gustos y prácticas culturales en general (Savage y Gayo, 2011; Bennett et al., 2009; Gayo et al., 2009) y ello convierte a los jóvenes, e igualmente a sus mayores, en algo más que transmisores de lo que aprendieron en sus hogares, y a la juventud en algo más que un período en la sombra de la vida personal.

Sin embargo, esta nueva atención a la edad por parte de los estudiosos interesados en la teoría del capital cultural, cuya naturaleza ha sido principalmente analítica. Es decir, dependiente de metodologías que han tratado de separar los efectos de las variables, ha llevado aparejada que no se haya atendido suficientemente a la desigualdad en cada uno de los diferentes momentos de la vida. Esto tiene consecuencias importantes, pues genera formas de ver al otro, en este caso al «joven», mucho más unitarias o generalizadas de lo que debiesen. Un claro ejemplo de ello puede ser el de los «jubilados». En general, podríamos entender que en Chile esta es una categoría social que incluye a personas con ingresos promedio inferiores a los de las personas que forman parte de la población activa. Ser jubilado en algunos países, como digo en promedio, puede llegar incluso a significar algo próximo a ser pobre o estar cercano estadísticamente a la pobreza. No obstante, cuando hacemos el análisis del comportamiento cultural de los jubilados distinguiendo por los ingresos del hogar y su nivel educativo nos encontramos con que estos son un grupo extraordinariamente diverso y, por ello, poco sentido tiene reconocerlos a todos por lo que hacen los que menos tienen entre ellos, incluso si este es su grupo más numeroso (Gayo et al., 2012).

Entendemos que idéntica es la situación de los jóvenes (Wilska y Pedrozo, 2007; Shildrick, 2006; De Castro, 2006; Lee, 2005; Bermúdez, 2001). Para resituarlos en el desarrollo teórico sobre el capital cultural es obviamente importante identificar cambios de acuerdo a la edad, pero no es suficiente. A este respecto, es sintomático que en un libro reciente y relevante en la disciplina, en el área de la sociología de la cultura, como Culture, Class, Distinction (Bennett et al., 2009), que 
estudia a la bourdieuana la formación del capital cultural en el Reino Unido, no haya ningún capítulo dedicado al tratamiento de la edad, ${ }^{3}$ a pesar de la relevancia que tiene en los análisis que en el mismo estudio se hacen. ${ }^{4}$ Es asimismo imprescindible aceptar que en este período hay algo peculiar que contribuye a la formación del capital cultural, pues de otro modo no se entendería que pudiesen encontrarse diferencias de gusto y práctica tan marcadas.

\section{LA TEORÍA DE LAS SUBCULTURAS: FORTALEZAS, INSUFICIENCIAS Y ALGUNAS POSIBLES SALIDAS}

Históricamente, una alternativa o complemento, según como se vea, a las teorías estructuralistas de las clases sociales ha sido lo que podemos denominar la teoría de las subculturas. ${ }^{6}$ Como núcleo teórico

3 En un importante estudio de Catalán y Sunkel (1990), que sirve como antecedente obligado de las investigaciones actuales sobre consumo cultural en Chile, es interesante observar como atienden preferentemente a variables socioeconómicas como los ingresos y la educación, omitiendo el tema de la edad.

4 En esta misma línea, y como parte del proyecto británico referido, puede verse Savage et al. (2005), Gayo-Cal et al. (2006), Savage y Gayo (2011). En todos estos estudios puede observarse como la edad aparece referida como una variable clave para la comprensión de la práctica cultural. Sobre Chile, con una aproximación de la misma naturaleza, véase Gayo et al. (2009).

5 Para Chile, evidencia detallada sobre diferencias entre grupos de edad con respecto a su participación cultural puede encontrarse en los informes elaborados por el Consejo Nacional de la Cultura y las Artes de este país para las encuestas de los años 2004-2005 (CNCA, 2007) y 2009 (CNCA, 2011). A partir de la primera de las encuestas mencionadas, la que une las realizadas en los años 2004 y 2005, se elaboraron artículos en los cuales se recogen algunas diferencias por cohortes de edad (Catalán y Torche, 2005). Asimismo, con información sobre el jefe de hogar, Bernasconi y Puentes (2001) ofrecen una descripción sobre el impacto de su edad sobre el consumo cultural de los hogares chilenos. Desde un punto de vista comparado y con material de entrevistas, puede consultarse los resultados de una investigación sobre consumo cultural en el Cono Sur de América, la cual incluyó los casos de Argentina, Chile y Uruguay (Gayo et al., 2011).

6 Mi relato es parcial en el tiempo y teóricamente. No obstante, creo hacer los énfasis que son necesarios para ayudar a entender en este artículo el 
tiene sus raíces en los estudios culturales británicos y en el giro cultural que reorientó fuertemente la agenda de las ciencias sociales al menos desde los años 60 del siglo pasado. En estas propuestas, había un genuino interés por entender las culturas populares o, en otros términos, la vivencia cotidiana de los subordinados o dominados en la sociedad. No en vano, las relaciones de clase tenían como base no sólo el dinero o la riqueza económica, sino las conductas y actitudes de aquellos que participaban en esta relación, fuese ello como «élite» o como «masa». Aquí no pretendemos, ni nos corresponde, reconstruir este pasado. Más bien, queremos hacer mención a su presente. Ello tiene como objetivo mostrar como lo que aquí entendemos como la «teoría de las subculturas», por un lado, ha mostrado tener una gran capacidad para describir prácticas culturales y estilos de vida, pero, por otro, se ha mostrado insuficiente para entender las bases estructurales sobre las cuales frecuentemente tales subculturas emergen. Esto no sólo se refiere al estudio e identificación de las variables que mayor peso tienen en la explicación de los patrones culturales, sino, y esto de forma igualmente destacada, a la visualización y comprensión de la posición relativa que los individuos tienen en los campos sociales, siendo en nuestro caso de particular interés el campo cultural. La atención al campo indica la relevancia que se otorga a la dotación relativa de capitales y al significado contextualizado de los comportamientos, pues finalmente el valor social o simbólico de lo que uno hace es fuertemente dependiente de lo que están haciendo las personas que están a su alrededor y que en conjunto contribuyen a formar el campo social de referencia.

Un amplio conjunto de estudios recientes, ${ }^{7}$ nos han ayudado a reconstruir los elementos propios de este enfoque teórico, al que aquí enfrentamos a algunos rasgos que entendemos característicos de la

trasfondo de mi argumento. Para una revisión acabada de la que aquí denomino como «teoría de las subculturas», en la cual podemos encontrar un posicionamiento bastante claro a favor de la idea de «subcultura», véase Muggleton (2005). Producto de una revisión crítica de este concepto es el libro editado por Bennett y Kahn-Harris (2004) After Subcultures, en cuyo capítulo introductorio se puede encontrar una reflexión muy completa sobre los antecedentes de la idea de subcultura, su uso por parte de la escuela británica de estudios culturales, y algunas alternativas conceptuales que han sido ofrecidas como solución a limitaciones de la idea mencionada.

7 Estos estudios son principalmente aquellos a los que hacemos referencia a lo largo de esta sección. 
teoría del capital cultural (ver tabla 1). La teoría a la que ahora atendemos adopta preferentemente una perspectiva «subcultural», y aquí me detendré en precisar el significado de este adjetivo, dado que tiene implicaciones tanto epistemológicas, como teóricas y metodológicas.

La «subculturalidad» se enmarca dentro de un paradigma que aquí denominaremos «posmoderno», pues los discursos son las nuevas estructuras que sustituyen el papel principal que en la modernidad tenían otras variables como los capitales. Para llegar a acceder y reconstruir esos discursos, adopta una visión «micro», cuya realización está asociada a los estudios de caso. Estos objetos de estudio podrían abordarse, medirse o tratarse de diferentes maneras, y aquí se apuesta por un enfoque subjetivista, lo que implica atender a motivaciones, actitudes, valores y afectos de los individuos que estamos estudiando. La metodología que ha sido entendida como la más adecuada para llegar al nivel discursivo ha sido la que habitualmente se denomina «cualitativa», en particular concretada de forma destacada en el uso de etnografías y observación participante, esto es, metodológicamente tienen una naturaleza etnográfica o próxima a la misma. Con ello se obtiene lo que podríamos entender como los «significados» de la acción, siendo el discurso una forma de la misma. Esta aproximación está asociada a una orientación teórica que enfatiza la descripción y no tanto la explicación, pues es el discurso lo que se trata de reconstruir y es el mismo el que tiene capacidad explicativa. Es decir, elevan la descripción de atributos o rasgos culturales al rango de una teoría. Una de sus principales fortalezas es su gran capacidad para «mapear» distinciones. Estas son de gran importancia, pues la definición de las comunidades o diferenciaciones se hará en torno a las prácticas (los discursos), lo que, en la medida en que se subraye únicamente la dimensión discursiva, dejará un rastro de transversalidad socioeconómica y, en sentido inverso, heterogeneidad de clase. Siendo una fortaleza su capacidad para identificar distinciones, una solución habitual de estos estudios es el recurso al etiquetaje, lo que transforma las concepciones individuales en intersubjetivas. A su vez, desde el punto de vista del interés en la estructura del gusto, encontramos - como ya indicábamos anteriormente de forma más genérica - una atención a la diferencia. Y si bien no es necesariamente así por razones lógicas, ha concentrado gran parte de sus fuerzas en entender la cultura popular, en manifestaciones muchas veces de comportamientos que frecuentemente han sido comprendidos como desviados. En coherencia con el hecho de que no se analiza el papel de las variables estructurales que 
puedan estar detrás de las prácticas, los cursos de acción aparecen como producto de decisiones individuales dentro de un contexto social particular. Ello abre la posibilidad del cambio tanto individual como colectivo desde un punto de vista diacrónico, pero, dado su énfasis en la comprensión de las distinciones discursivas, termina promoviendo la continuidad, pues a menudo encuentra valor en las visiones informadas por la subordinación y la resistencia.

TABLA 1: RASGOS DE LA TEORÍA DEL CAPITAL CULTURAL FRENTE A LA TEORÍA DE LAS SUBCULTURAS

\begin{tabular}{|c|c|c|}
\hline & T. CAPITAL CULTURAL & T. SUBCULTURA \\
\hline Paradigma & Modernidad & Posmodernidad \\
\hline Visión & $\begin{array}{l}\text { Holística o «sistémica» } \\
\text { (macro) }\end{array}$ & Estudio de caso (micro) \\
\hline Enfoque & Estructuralismo & Subjetivismo \\
\hline Metodología & $\begin{array}{l}\text { Cuantitativa y cualitativa } \\
\text { (entrevistas) }\end{array}$ & $\begin{array}{l}\text { Cualitativa (etnografía, } \\
\text { observación participante) }\end{array}$ \\
\hline Obtiene & Variables explicativas & Significados \\
\hline Orientación teórica & Explicación & Descripción \\
\hline Fortalezas & Explicar desigualdades & Mapear distinciones \\
\hline Comunidad & Clases o unidad & $\begin{array}{l}\text { Unidad en torno a las } \\
\text { prácticas: transversalidad } \\
\text { socioeconómica y hetero- } \\
\text { geneidad de clase }\end{array}$ \\
\hline Solución & Gradualidad & Etiquetaje \\
\hline Estructura gusto & $\begin{array}{l}\text { Desigualdad económica y } \\
\text { cultural }\end{array}$ & Diferencia \\
\hline Tipo de cultura & Alta cultura & Cultura popular \\
\hline Curso de acción & $\begin{array}{l}\text { Estructuralmente } \\
\text { condicionado }\end{array}$ & $\begin{array}{l}\text { Decisión individual } \\
\text { dentro de un contexto } \\
\text { social particular }\end{array}$ \\
\hline $\begin{array}{l}\text { Perspectiva } \\
\text { diacrónica }\end{array}$ & $\begin{array}{l}\text { Continuidad (reproducción de } \\
\text { las posiciones en el espacio } \\
\text { social) }\end{array}$ & $\begin{array}{l}\text { Cambio } \\
\text { (historia colectiva) }\end{array}$ \\
\hline Promueve & Cambio & Continuidad \\
\hline
\end{tabular}

Los intereses de los estudios sobre los jóvenes realizados desde la teoría de las subculturas han venido variando, pero aquí haremos mención a tres, alguno de los cuales veremos en los datos que fueron recogidos para el caso analizado en este artículo, la juventud en Chile. El primero de ellos —el cual ya tiene una larga historia y ha sido cen-

8 Esta idea la incorporé a partir del trabajo de Wel et al. (2008). 
tral en el desenvolvimiento de los estudios culturales - se relaciona con el estudio de la cultura de la clase obrera, en las formas que esta pueda adoptar. A menudo, se trata de atender a patrones culturales cuya práctica parece estar asociada a una resistencia al aprendizaje o la normalización en la escuela y que, por tanto, lleva a la exclusión o la subordinación social (Wel et al., 2008; Terpstra, 2006; Willis, 1977). El segundo tema es el uso de las tecnologías, bien sean estos los medios de comunicación que han hecho su aparición sobre todo desde la segunda mitad del siglo XX, de un modo destacado la televisión, bien se trate del uso de instrumentos tecnológicos en la vida cotidiana como lectores y grabadores de música, plataformas de juegos o el propio internet (Vanden Abeele y Roe, 2011; Hagen, 2007; Wilska y Pedrozo, 2007; Lee, 2005). En tercer lugar, una temática recurrente ha sido la de la música, debido a su fuerza para articular grupos de personas en torno a los estilos de vestimenta, las formas de pensamiento y la ocupación de lugares (Kiilakoski y Oksanen, 2011; Selfhout et al., 2008; Baker y Cohen, 2008; Toth, 2008; Bermúdez, 2001). No en vano, si combinamos estas temáticas, obtenemos un perfil que ha llegado a ser prototípico de los jóvenes, pues son muy frecuentemente descritos como contestatarios (en la escuela), tecnológicos y «musicofílicos».

Teniendo ambas aproximaciones teóricas fortalezas y debilidades, la posibilidad de aproximar o hacer confluir ambas teorías puede contribuir a avanzar en una explicación cabal de la participación cultural de los jóvenes. En realidad, esto no es ninguna novedad y ya ha sido intentado, aunque esa fusión creemos que es insatisfactoria. ¿Cómo se ha propuesto fundir ambas teorías y por qué estamos insatisfechos? La respuesta a la primera pregunta se refiere al intento de transformar las subculturas, cualquiera que sea, en capital. Ahora ya no se trataría de un «capital cultural» sino de un «capital subcultural» (Leonard, 2008; Jensen, 2006). ${ }^{9}$ En un sentido similar, desde estudios interesados en mantener el vínculo entre el capital cultural y las subculturas, han habido respuestas a la necesidad de relacionar las últimas con las variables estructurales que contribuyen a definirlas y que son expresadas a través de ellas (Jensen, 2006). Sin embargo, esta solución expresa un relativismo en relación a lo que consideraríamos capital, lo que nos llevaría a situaciones que los individuos vivirían muy frecuentemente como contradictorias, pues una práctica o símbolo podría ser un capital en nuestro barrio depauperado y una seria limita-

9 En este punto, la autora se refiere a la contribución de Sarah Thornton. 
ción cuando salimos de sus fronteras. Quizás este es un riesgo presente en la idea de campo social, o en usos posibles de la misma, y debemos ser conscientes de ello.

\section{DATOS Y METODOLOGÍA}

El encuentro de las teorías del capital cultural y de las subculturas no tiene como único lugar posible una nueva teoría, como fusión combinada de ambas. Aunque menos explorado, también es importante referirse a las posibilidades que abre la confluencia al nivel de los métodos. Ello significa rechazar que el único modo de obtener información sobre los significados es el etnográfico y que las encuestas recogen datos un tanto superficiales sobre los individuos desde el punto de vista de su subjetividad, pues ello parece más un relato exagerado y cargado de prejuicios que una realidad necesaria.

Reconociendo el aporte de los estudios más etnográficos vinculados a la teoría de las subculturas, e igualmente la contribución de las aproximaciones metodológicas más próximas a la teoría del capital cultural, en este trabajo nos inclinamos por la segunda, si bien tratando de poner el énfasis en la conveniencia de vincular a este nivel ambas teorías. No obstante, en este caso, atendemos a lo que el enfoque de las subculturas no ha alcanzado a hacer convenientemente. Es decir, analizamos la práctica cultural desde el punto de vista de las variables que la estructuran, ${ }^{10}$ y agregamos algo en lo que tal perspectiva ha sido destacada, el uso de información cualitativa. A continuación detallamos las características de los datos analizados.

La naturaleza de los datos y el contenido de las preguntas en las encuestas que se han realizado hasta la fecha en Chile dificultan que

10 A este respecto, en coherencia con lo que aquí sostenemos, Bolin afirma que «As a consequence of this identity focus, and the ethnographic methods used, youth cultural studies ... have tended to favour a micro level analysis where individual interpretations of media texts have been highlighted, often in terms of pleasure, resistance, empowerment etc., which have mostly been studied from the perspective of the individual, i.e. which identities he or she comes up with as an end product. Few studies have taken the analysis further and linked this micro level analysis to a broader focus at macro levels; for instance linking identity production to the structural level of cultural forms, and how these cultural forms in turn deeply affect the possibilities at hand for the individual to construct his or her identity» (1999:50). 
avancemos empíricamente hacia una aproximación más integrada de las teorías antes mencionadas. Con los datos con los que contamos, no obstante, es posible explorar hipótesis provenientes de la teoría del capital cultural ya explicitadas en los apartados previos.

Este estudio combina dos tipos de datos, los de la encuesta del Instituto Nacional de la Juventud (INJUV) realizada en el año 2009, la última disponible hasta la fecha, y los resultados provenientes de un conjunto de cuatro focus groups realizados durante el año 2007 en la ciudad de Santiago de Chile. Por lo que respecta a la encuesta, tiene una cobertura territorial nacional, fue realizada a jóvenes de edades comprendidas entre los quince y los veintinueve años, en zonas rurales y urbanas, y el número de personas encuestadas ascendió a siete mil setenta, todas las cuales fueron entrevistadas cara a cara. El cuestionario no se dedica de forma exclusiva o principalmente al consumo cultural, pero contiene información valiosa sobre esta temática, cuyo análisis ha servido para desarrollar este estudio. En el análisis de los datos se utilizó la técnica estadística conocida como análisis de correspondencias múltiples — que Bourdieu contribuyó decisivamente a popularizar en las ciencias sociales-y en los últimos años ha tenido una gran expansión en los estudios informados por la teoría del capital cultural (Savage y Gayo, 2011; Bennett et al., 2009; Gayo et al., 2009; entre muchos otros).

Esta técnica estadística ha sido frecuentemente relacionada con el gráfico que tiene asociada a la producción de sus resultados. La misma tiene dos objetivos principales. Por un lado, nos permite ver la asociación relativa entre las categorías de un conjunto de variables categóricas, para lo cual podemos recurrir como referencia a la distancia entre las mismas que podemos visualizar en los gráficos producidos. Como regla de interpretación, podemos decir que entre dos categorías cualesquiera que son comparadas con respecto a una tercera, la mayor cercanía a una de las dos primeras significa que las personas con ese rasgo realizan en un porcentaje mayor la actividad (de la categoría de referencia o comparación) que las personas de la segunda categoría. Las actividades que están presentes en un lugar próximo en el espacio se entenderá que están positivamente asociadas; es decir, que los individuos que hacen una muy probablemente harán las otras con similar posición espacial. Además de esto, el análisis de correspondencias múltiples puede ser entendido como una técnica apropiada para la identificación de variables latentes. Ello quiere decir que nos permite estudiar la estructura que está detrás de las diferencias anteriormente 
observadas. En la obra de Bourdieu (1979), se destacan los capitales como elementos centrales de esa estructura, pero eventualmente puede haber otras variables relevantes, dependiendo de nuestro objeto de estudio. En nuestro caso, abordamos esta materia más adelante.

En lo que se refiere a los focus groups, se realizaron ocho, y cuatro que incluían a personas dentro del rango etario 18-40. Cada uno de ellos se refirió a un grupo socioeconómico, siendo incluidos los siguientes: C1, C2, C3 y D. ${ }^{11}$ Este trabajo cualitativo se hizo dentro del marco de un proyecto dedicado a explorar las desigualdades en el consumo cultural de los chilenos, ${ }^{12}$ para lo cual se recurrió tanto a criterios socioeconómicos como etarios. Distinciones de género pueden ser igualmente percibidas. Los focus groups fueron heterogéneos desde el punto de vista del sexo. Cada uno de ellos tuvo la participación de unas ocho personas.

\section{EL ESTUDIO EMPÍRICO DESDE LA PERSPECTIVA DEL ANÁLISIS DEL CAMPO SOCIAL}

Estudiar la participación cultural adoptando la perspectiva del campo social tiene al menos cuatro objetivos. El primero tiene que ver con el interés por el estudio de la desigualdad. Es decir, es una aproximación atenta a las asimetrías en términos de recursos, principalmente los económicos y culturales, pero puede haber otros, como sería el caso por ejemplo del género. En segundo lugar, implica sospechar, hipote-

11 Generalmente, el grupo socioeconómico se calcula en base a la capacidad de consumo, o una combinación de consumo y educación, si bien otras alternativas son posibles. Los cálculos se hacen en base a datos de encuesta, es decir, a partir de las respuestas de cada uno de los encuestados. Siendo el nuestro un estudio cualitativo, y no pudiendo hacer ese tipo de cálculos a priori para la selección de las personas, se externalizó el reclutamiento de los participantes de los focus groups, y la decisión fue adoptada por una empresa dedicada a este tipo de actividades. Para ello utilizan criterios como profesión, lugar de residencia, educación, ingresos, los cuales generan resultados aproximados. En cualquier caso, una vez hecho el estudio, pudo verificarse que efectivamente había diferencias importantes, tal y como se esperaría, lo que indica que la estrategia servía para diferenciar de manera conveniente y verosímil.

12 Proyecto Semilla financiado por la Universidad Diego Portales (Santiago, Chile), entre los años 2007 y 2008, y titulado «Capital cultural: producción, configuración y efectos». 
tizar o entender que las desigualdades desde el punto de vista de una práctica suelen reproducirse en otras de forma coherente, lo que es una versión de la célebre hipótesis de la homología de las prácticas culturales, detrás de la cual, a la bourdieuana, estaría el habitus. En tercer lugar, supone adoptar un enfoque relacional, en el sentido de entender que lo que cada persona hace, o el conjunto de las actividades en las que participa, o el conjunto de gustos que manifiesta, tiene sentido dentro de la estructura creada por la interacción entre todas las personas que participan de este «juego». Finalmente, en cuarto lugar, las desigualdades o diferencias presentes en la participación de los sujetos están íntimamente relacionadas con disputas por el poder en el campo, a través de las cuales se producen y reproducen las asimetrías presentes en un momento histórico determinado.

Con el objeto de estudiar y visualizar la participación cultural de los jóvenes chilenos desde una perspectiva de análisis del campo social, los siguientes dos apartados los dedicamos a la presentación de los resultados producto del estudio de la encuesta del INJUV, haciendo reconstrucciones del campo mediante el uso de variables de temas diferentes y explorando la estructura de cada una de las soluciones. Además, en la sección siguiente, exponemos los resultados provenientes del análisis de los focus groups y estudiamos en qué medida nos permiten dialogar con los que provienen del análisis de la encuesta.

\section{a) El espacio social de la participación cultural de los jóvenes}

La encuesta del INJUV no está diseñada para recoger información principalmente sobre participación cultural. El cuestionario utilizado contiene una amplia serie de preguntas que abarcan muchas dimensiones de la vida de los jóvenes. Por lo tanto, su uso para nuestros propósitos impone hacer una selección de las variables que mejor encajan con el estudio de la práctica cultural. En este caso, tres han sido las temáticas sobre las cuales hemos encontrado información: la participación en organizaciones, las actividades culturales realizadas y el uso de internet. Debido a que generalmente a los encuestados les preguntan en primer lugar si tienen alguna relación con las cuestiones que les van a plantear (v. g., si usa internet antes de preguntarles sobre los tipos de empleo del mismo), se hace técnicamente difícil hacer una representación conjunta de todas las variables, y es por ello que presentamos cada uno de los temas en distintos gráficos, aunque el tratamiento quiere integrar todos los hallazgos. 
Por lo que respecta a la participación en organizaciones culturales, el gráfico 1 claramente muestra diferencias importantes. La primera distinción que observamos, asociada al primer eje, es la que separa a los que participan de los que no lo hacen. Algunos jóvenes son más participativos que otros. Además, los que no forman parte de organizaciones, tienden a no serlo de ninguna. Por su parte, y en un sentido diferente, hay un grupo de jóvenes relativamente participativos, pero en este caso están divididos. Por un lado, encontramos a los que forman parte de barras de fútbol y clubes deportivos. Por otro lado, están los grupos scouts. Finalmente, encontramos una importante asociación entre la pertenencia a tribus urbanas, el involucramiento en comunidades virtuales, la participación en grupos de arte y el contacto con grupos reunidos en torno a pasatiempos.

\section{GRÁFICO 1: LA PARTICIPACIÓN EN ORGANIZACIONES}

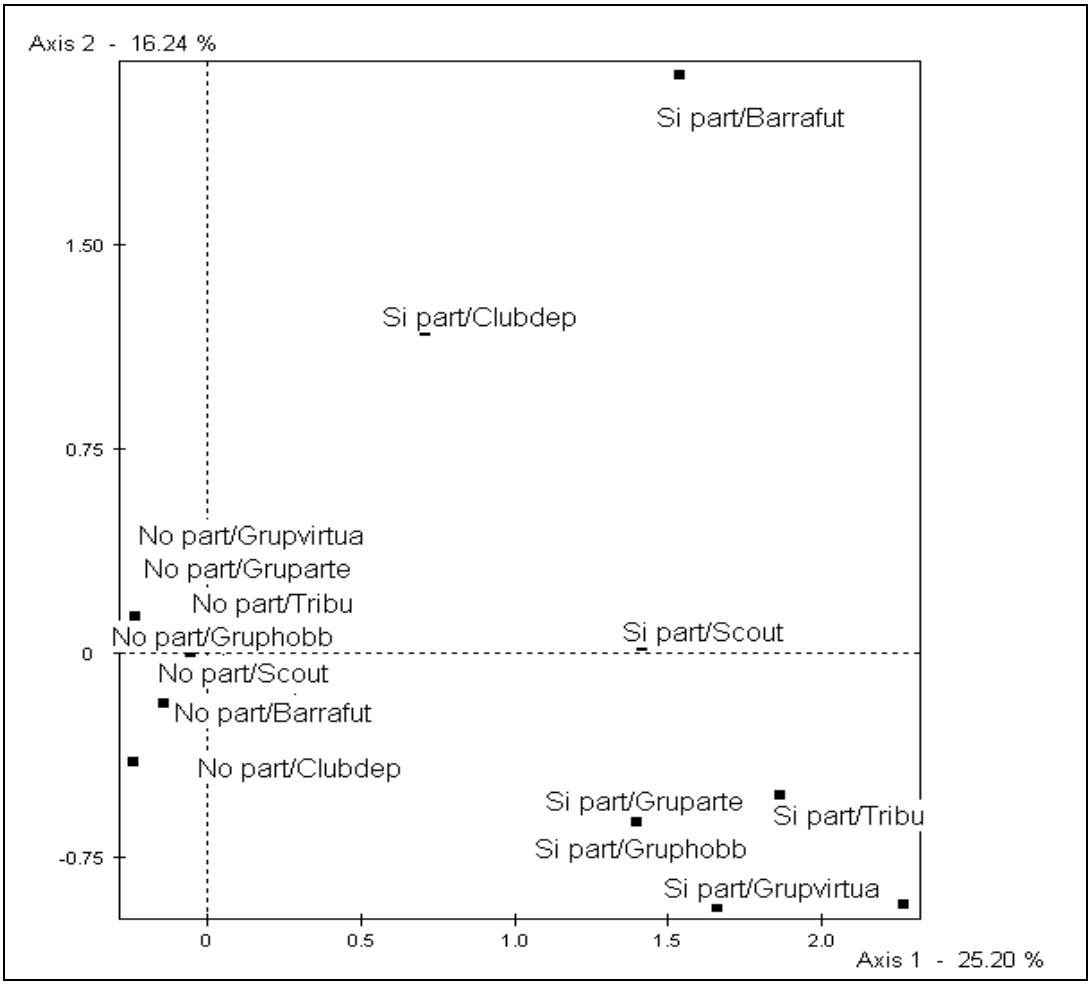

Fuente: Encuesta INJUV 2009. 
Si atendemos a las actividades culturales realizadas, también encontramos una importante diferencia entre unos jóvenes y otros (ver gráfico 2).

GRÁFICO 2: LAS ACTIVIDADES REALIZADAS

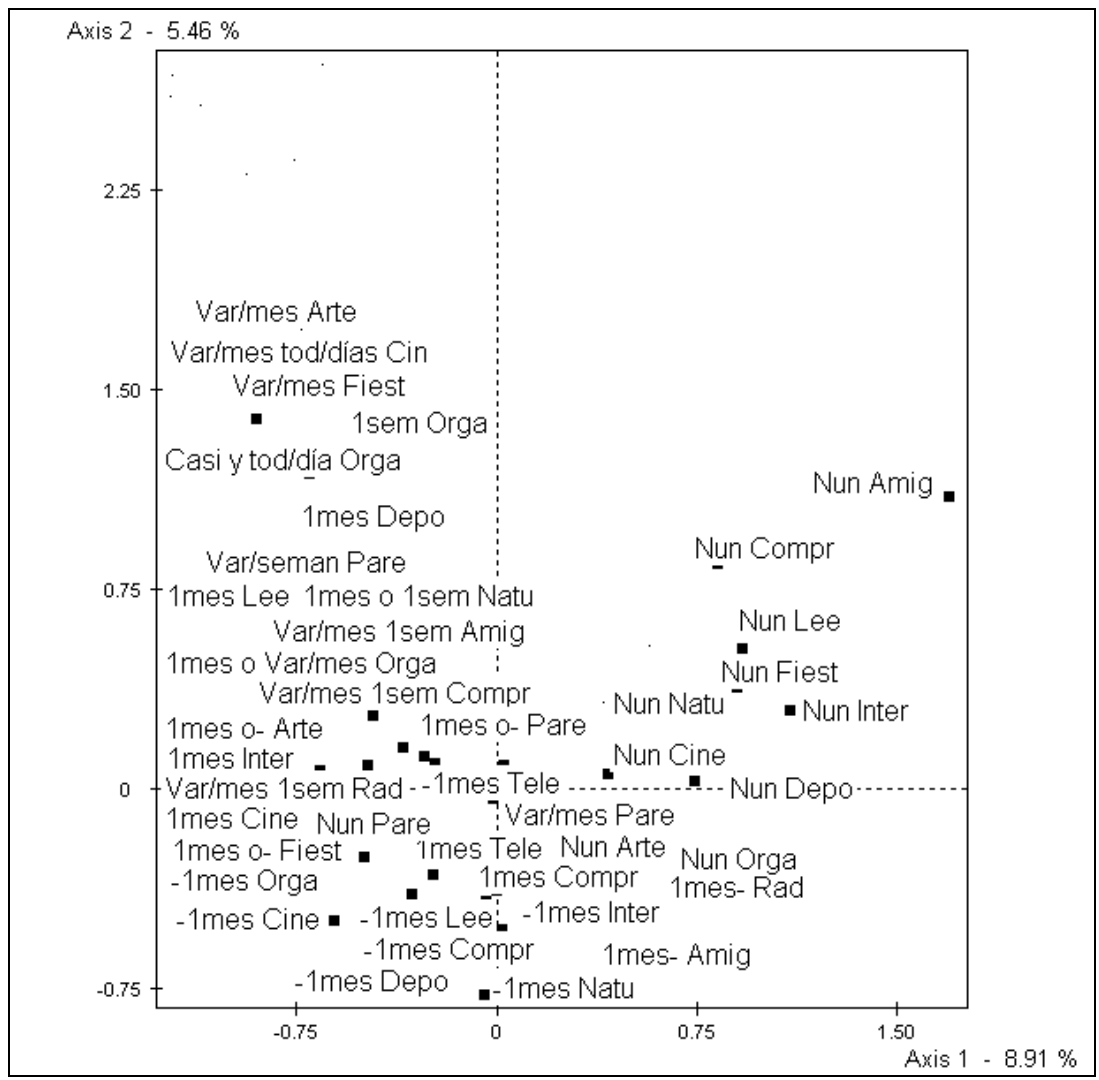

Fuente: Encuesta INJUV 2009.

Asimismo, observamos igualmente que la frecuencia de participación en las diferentes actividades está asociada positivamente. En otras palabras, los que manifiestan que nunca salen a fiestas, tampoco leen, ni salen a disfrutar de la naturaleza, ni navegan por internet, ni van al cine, ni practican deporte alguno. En sentido contrario, hay jóvenes que dicen estar involucrados frecuentemente en la realización de actividades artísticas, van al cine en forma constante, participan habitualmente en organizaciones, practican deportes cotidianamente, suelen estar con su pare- 
ja, leen, salen de compras y se reúnen recurrentemente con amistades. Aquí, la asociación, más que por tipo de actividad, es por frecuencia. A mayor participación en una actividad, mayor lo será en las demás. No cabe duda que esto abre una brecha entre jóvenes activos y pasivos, lo que muestra que la juventud no es vivida por todos por igual.

$\mathrm{Si}$ a algo se asocian los jóvenes, no cabe duda, que es con las nuevas tecnologías. Normalmente pensamos en ellos como sus usuarios por antonomasia, como si se tratase de un código que pertenece principalmente a los que lo incorporaron en su infancia, una especie de nueva alfabetización a la que los adultos les resulta difícil incorporarse. Sin embargo, las diferencias entre los jóvenes también son muy notables (ver gráfico 3). En otras palabras, no todos ellos son igualmente «tecnológicos». Al igual que sucedía con las actividades culturales, observamos una fuerte relación entre las frecuencias de los diferentes usos de internet: cuanto más es utilizado para un propósito, mayor es su empleo con otros objetivos. Esto significa que más que tipos de usuarios definidos de acuerdo a los usos, lo que encontramos son perfiles de aquellos dependientes de la frecuencia de su utilización. En síntesis, los que emplean internet para buscar información, lo hacen también para bajar programas, comprar, leer prensa y revistas, chatear, conseguir y escuchar música, entre otros. Y en el caso de los que lo hacen en menor medida, su menor uso tiende a ser transversal a las diversas formas de apropiación de la tecnología de internet.

Análisis previos sobre el gusto y la participación cultural en Chile habían concluido que los jóvenes tenían un comportamiento característico (Gayo et al., 2009). Mostraban una propensión mayor que todos los demás al activismo cultural, pensándolo desde el punto de vista de ser protagonistas de las actividades mismas y no como observadores. Los jóvenes hacen teatro, tocan instrumentos y bailan, y ello en una medida superior a la de todos los demás grupos etarios. Por eso, en el trabajo que aquí citamos se les denominó «hacedores» culturales. Sin embargo, si bien se reconoció que la intensidad participativa variaba de acuerdo con los recursos económicos y culturales disponibles, las diferencias dentro de la juventud no han sido suficientemente exploradas en la literatura informada por la teoría del capital cultural. Aquí dejamos constancia de que este hecho tiene una envergadura que exige atenderlo convenientemente. En todo caso, esto es sólo la primera parte del análisis, pues la segunda requiere que nos preguntemos por las variables que pueden estar detrás de tal diversidad. A ello nos dedicamos en la sección siguiente. 
GRÁFICO 3: EL USO DE INTERNET

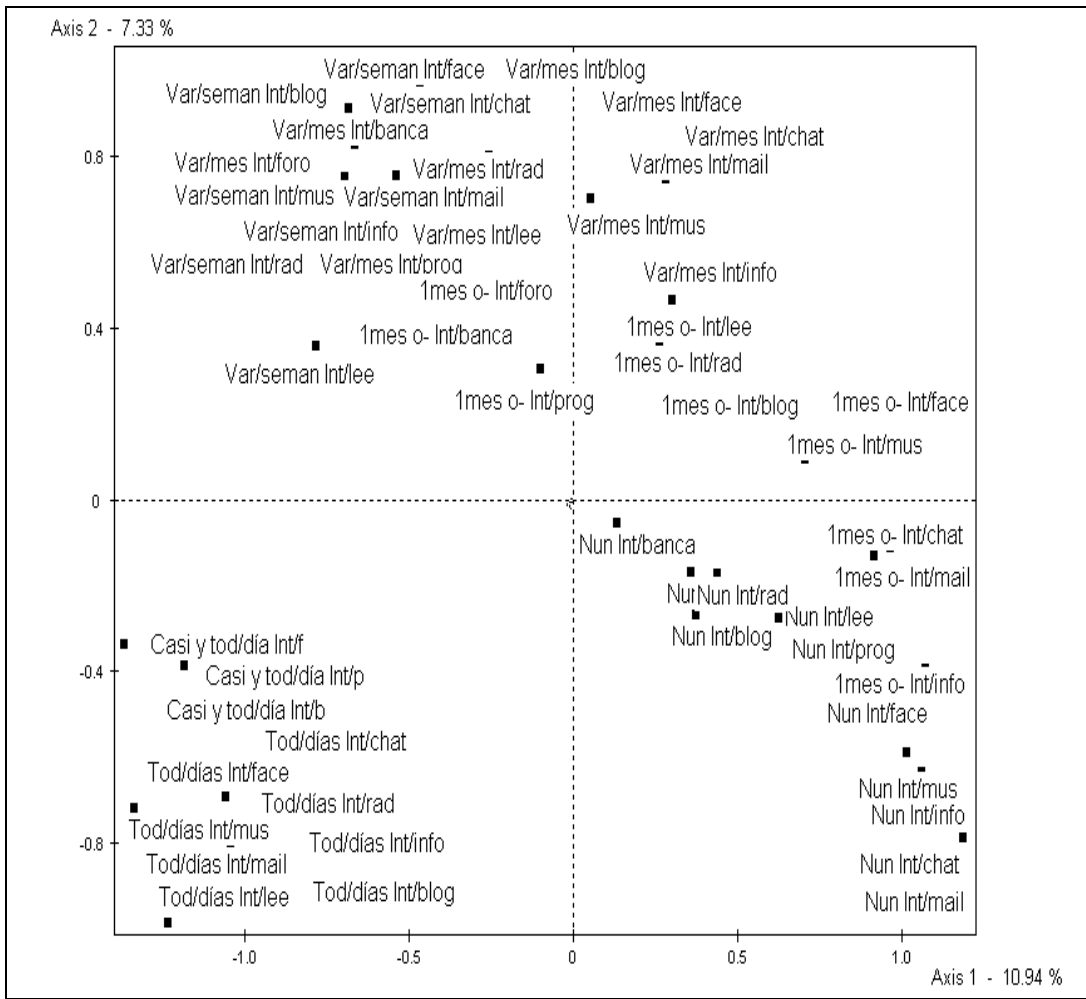

Fuente: Encuesta INJUV 2009.

\section{b) Estructura del espacio social}

Una vez que hemos descrito cómo se distribuyen las categorías de las variables en el espacio, y habiendo constatado que hay relaciones evidentes entre ellas, el análisis de correspondencias múltiples nos permite explorar qué variables pudieran estar asociadas con cada una de las dimensiones, o ejes. En este caso, estudiaremos los dos primeros, pues es un procedimiento habitual en estas aproximaciones al ser los que tienen una mayor capacidad explicativa en términos de varianza estadística. Para la presentación de los resultados, recurriremos al orden de la sección anterior, comenzando con la participación en organizaciones y finalizando con el uso de internet. Es importante entender que los gráficos de los espacios sociales referidos a la misma temática son idénticos; 
es decir, a modo de ejemplo, el estudio de la estructura del gráfico construido en base a la realización de actividades culturales se hace a través de un gráfico del mismo espacio al cual se le han superpuesto variables que el investigador considera que pudieran estar asociadas a la dispersión de categorías observada. En definitiva, son dos gráficos por razones de visualización de los hallazgos, pero es el mismo espacio.

GRÁFICO 4: LA ESTRUCTURA DE LA PARTICIPACIÓN EN ORGANIZACIONES

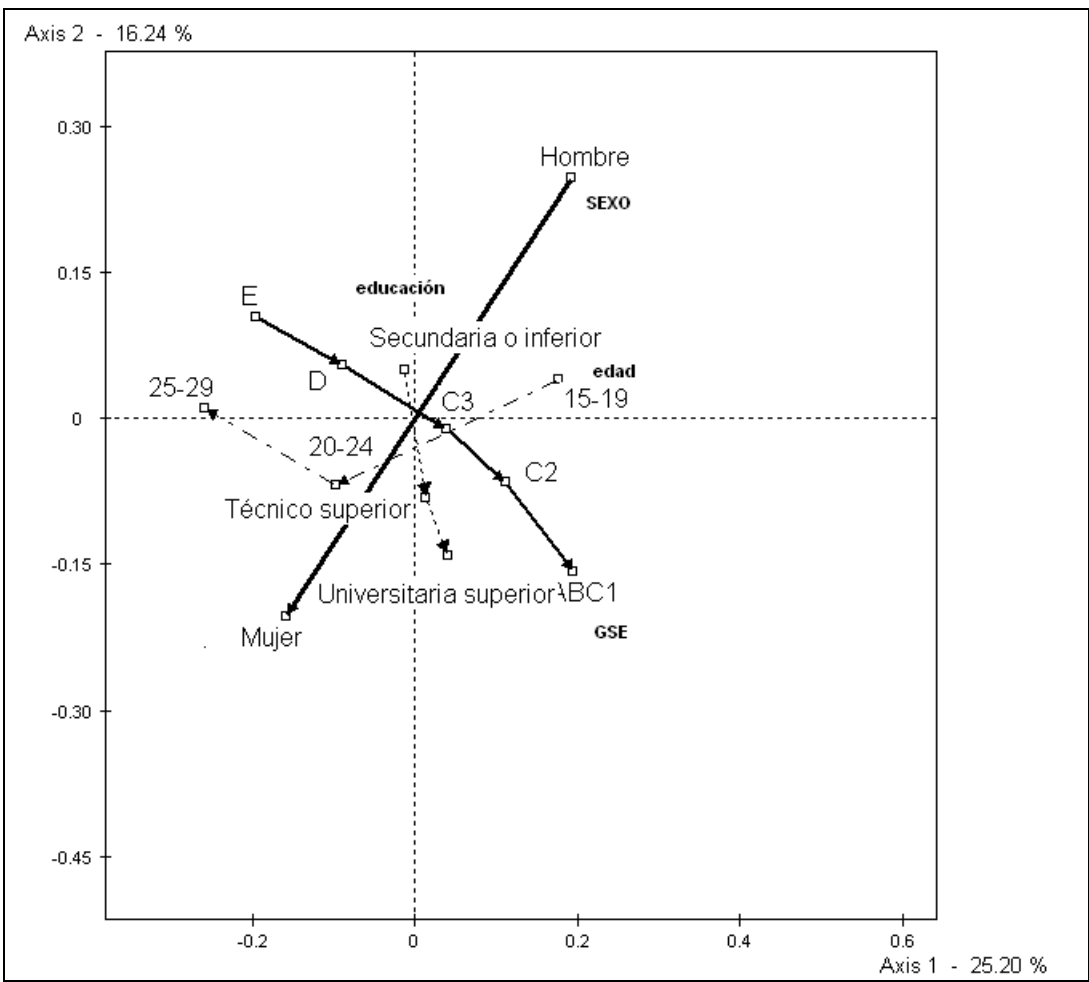

Fuente: Encuesta INJUV 2009.

En primer lugar, por lo que respecta a la participación en organizaciones culturales, se observa un espacio con una estructura compleja (ver gráfico 4). En principio, tal y como esperaríamos de acuerdo con la teoría del capital cultural, los que participan muestran una mayor acumulación de capitales, tanto económicos ( $\mathrm{ABC} 1)$ como culturales (estudios universitarios), pero el espacio tiene una complejidad que obliga a ir más allá de esta explicación. Y ello es así por varias razo- 
nes. Primero, porque los recursos económicos y culturales nos ayudan a entender principalmente un tipo de participación organizativa, la que se refiere al involucramiento en grupos de arte, en tribus urbanas, comunidades virtuales y grupos organizados en torno a sus pasatiempos. Segundo, porque la edad también muestra tener una gran relevancia. La juventud, definida en la encuesta como la edad comprendida entre los quince y los veintinueve años, contiene un rango de edades dentro del cual hay una notable diversidad. A este respecto, los más jóvenes entre los jóvenes son los más participativos, lo que muestra que el muchas veces mentado alargamiento de la juventud no oculta que importantes cambios se producen en lapsos de pocos años. Tercero, porque las diferencias de género son muy relevantes. Las mujeres y los hombres hacen frecuentemente cosas distintas. Las primeras parecen participar un poco menos que los segundos, lo que podría plantear cuestiones sobre la oferta participativa existente, quizás «masculino-céntrica», y cuando lo hacen, los grupos de deporte son dominados de forma dramática por el género masculino, quizás porque las identidades de los hombres están fuertemente ancladas en actividades de esta naturaleza.

GRÁFICO 5: LA ESTRUCTURA DE LAS ACTIVIDADES REALIZADAS

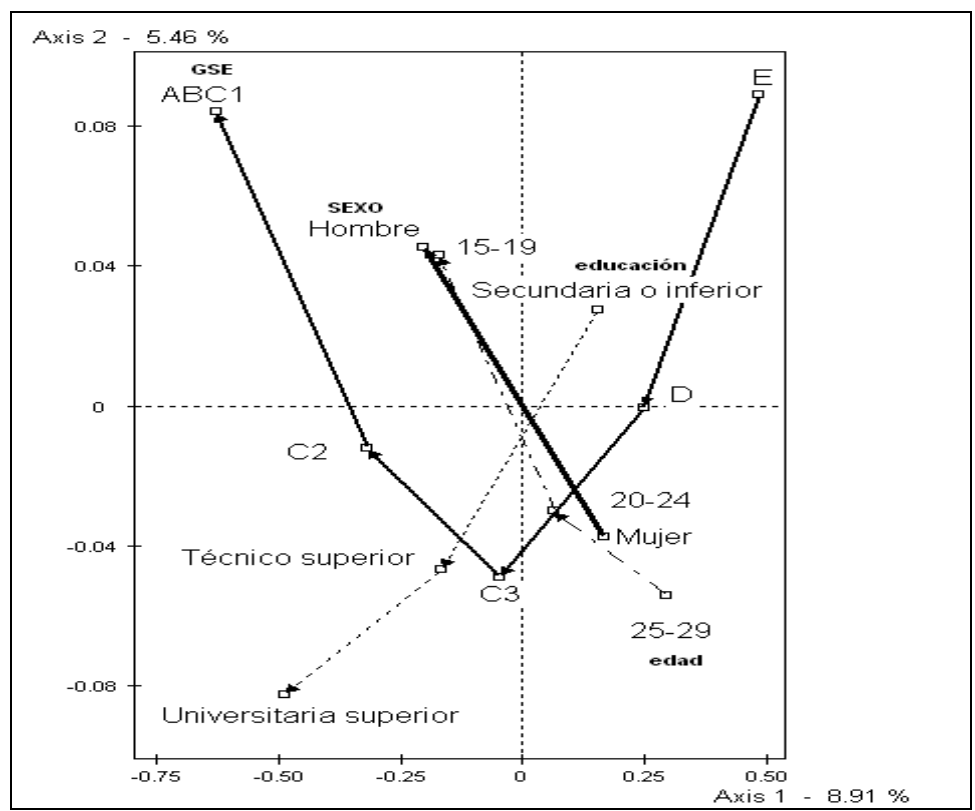

Fuente: Encuesta INJUV 2009. 
Si atendemos al gráfico relativo a la participación en actividades culturales (ver gráfico 5), nuevamente la estructura del espacio social se ve fuertemente afectada por los capitales, de forma destacada por el grupo socioeconómico, cuya trayectoria tiene la misma forma de la nube de categorías representada en el gráfico asociado (el gráfico 2). Eso significa que cuanto mayor es la acumulación de capital económico, mayor será la participación promedio de las personas. Un patrón similar muestra la asociación con la educación, pero esta pareciera enfrentar la participación a la no participación, siendo los niveles más altos del activismo asegurados por un nivel elevado de recursos económicos, quizás condición de un perfil participativo que muestra una alta intensidad de involucramiento cultural. Por su parte, la edad y el género muestran efectos menos claros, si bien una menor edad y ser hombre parecieran ser categorías más próximas a la participación que las otras que complementan estas variables.

GRÁFICO 6: LA ESTRUCTURA DEL USO DE INTERNET

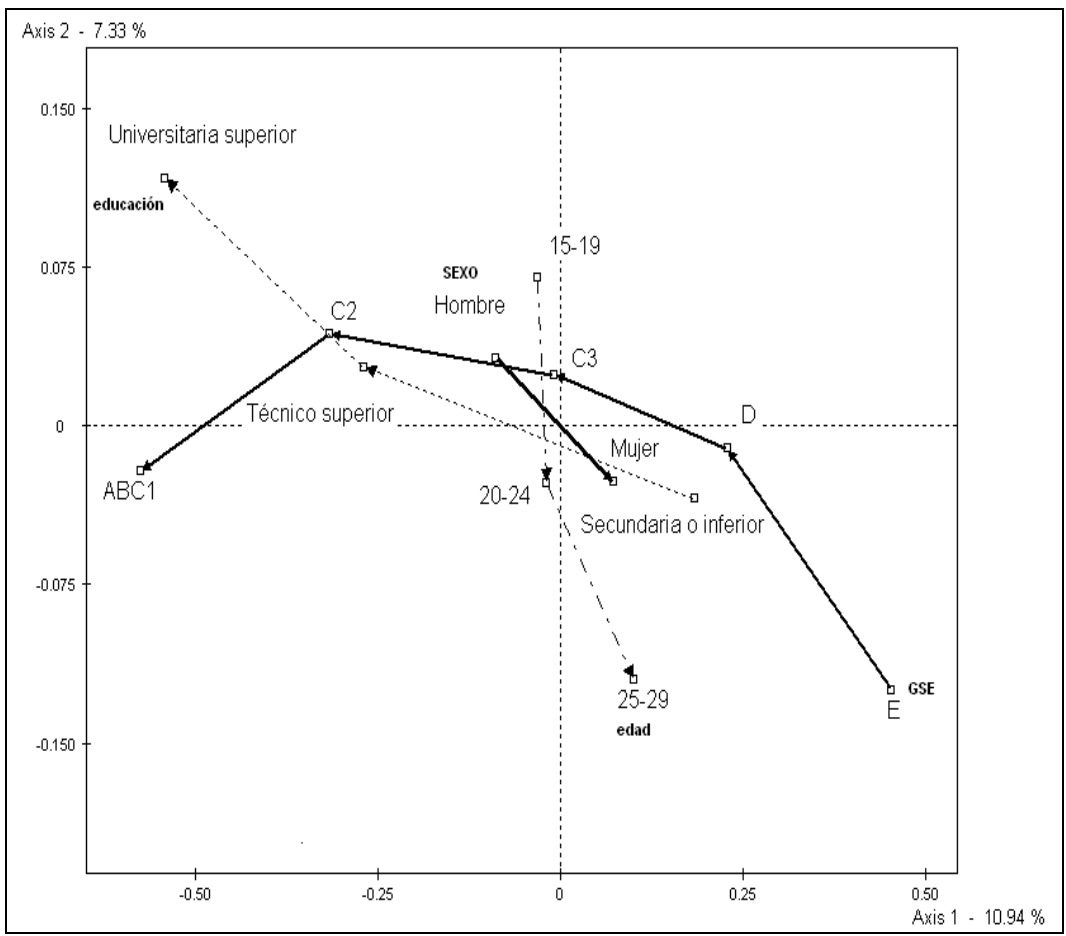

Fuente: Encuesta INJUV 2009. 
En tercer lugar, en lo que afecta al uso de internet (ver gráfico 6) este está profundamente influido por el grupo socioeconómico de pertenencia. Es así que se observa que los que más capacidad económica tienen (ABC1) manifiestan tener una intensidad de empleo de internet mucho más elevada que los que menos recursos han acumulado (E). Puede ser que el digital gap se haya ido estrechando históricamente, pero ha existido y estas mediciones, no muy lejanas, nos proporcionan evidencia sobre su probable presencia en la actualidad. En este caso, la educación tiene un comportamiento muy similar al del gráfico anterior, esto es, a mayor nivel educativo, mayor uso tenemos de internet, pero los niveles más elevados serían únicamente asegurados por una notable acumulación de capital económico. De un modo similar, también en este caso la edad y el género, tendrían un impacto menor, aunque, como ya comentamos para el caso de las actividades culturales, los jóvenes de menor edad (aquí definido como el grupo 15-19) y los hombres parecen mostrar una inclinación mayor a convertirse en «internautas» frecuentes.

\section{LAS PRÁCTICAS DESDE UN ESTUDIO DE FOCUS GROUPS}

La aproximación del análisis de campo al estudio de las actividades de los jóvenes chilenos nos ha servido para mostrar, por un lado, como el activismo cultural es transversal al diferente tipo de actividades, esto es, el que hace una tiende a hacer las otras, y frente a este perfil participativo estaría, en el otro extremo, un patrón de pasividad cultural. Por otro lado, hemos comprobado que la participación cultural está fuertemente influida por la acumulación de los recursos económicos y culturales, y también, aunque en menor medida, por la edad y el género. Sin embargo, poco sabemos sobre la cotidianeidad de estas personas, sobre el marco dentro del cual realizan estas actividades, y sobre las formas de participación cultural que ellos más destacan. Para aportar información que permita contribuir a cubrir esta carencia, hemos analizado un grupo de cuatro focus groups compuestos por personas con edades comprendidas entre los dieciocho y los cuarenta años. Es obvio que el encaje de edades no es perfecto, pero dado que hay un grado importante de solapamiento en las mismas y que los focus groups se aplicaron a cuatro grupos de personas homogéneos - desde el punto de vista de su extracción socioeconómica - y siendo esa la variable que mayor impacto ha mostrado, la información recogida en años similares (2007, focus groups y 2009, la encuesta) puede ofrecernos patrones cuya vinculación debiera fortalecer y por ello mejorar 
nuestro conocimiento tanto del contenido cultural de las posiciones dentro del espacio social, como de las realidades socioeconómica y sociodemográfica asociadas con las mismas. El análisis que a continuación presentamos tiene ese objetivo. Los siguientes puntos exponen los perfiles tan acabados como nos ha permitido el material recogido, separando los mismos por una combinación de grupo socioeconómico y género; es decir, haremos referencia a los patrones de las mujeres y hombres $\mathrm{C} 1$ por separado, y continuaremos del mismo modo con los siguientes grupos de acuerdo al mismo orden de género y por nivel socioeconómico de mayor a menor. Adicionalmente, son intercaladas algunas citas al final de la descripción de varios de los perfiles como una muestra más vívida de las intervenciones de las personas participantes sobre las que aquellos fueron elaborados.

a) Mujeres $C 1$. Su tiempo libre es asociado a aprovechar la familia y las amistades. Salen frecuentemente la noche de los sábados a cenar con su pareja. Son habituales de los cines, los cafés conciertos, los recitales, y de espectáculos en general. En ocasiones practican actividades como estudiar canto lírico. Ven a menudo programas culturales en la televisión, y a veces se inclinan por las películas antiguas. Cuando hablan de cine, manifiestan un gusto por las grandes producciones (Titanic, Star Wars, Harry Potter, el Señor de los Anillos). Sin embargo, el cine chileno lo encuentran malo, vulgar y repleto de expresiones soeces. Viajan dentro de Chile y a Argentina, y suelen comprar libros en este último país.

b) Hombres C1. Su tiempo libre lo dedican preferentemente a sus hijos y, más en general, a su familia. Van al mall (centro comercial). Descansar lo asocian a compartir con sus amigos y polola (novia). Juegan a la Wii con su pareja. Salen a comer, para lo cual prefieren cambiar de destino con frecuencia, pues ello les permite tener un conocimiento más amplio de lugares. A este respecto, mencionan emplazamientos exclusivos de la capital, como Isidora Goyenechea y Vitacura. Van al cine o arriendan películas. Les gustan especialmente las de acción, los dramas y las comedias, mostrando rechazo por la ciencia ficción. Se manifiestan a favor de las grandes producciones cinematográficas, para verlas juzgan ideales las condiciones que ofrecen los cines debido a su calidad de imagen y sonido. Consideran malo el cine chileno, pues dicen no sentirse representados por el mismo, al que tildan de marginal y politizado. Van al estadio (de fútbol). Ven la televisión, prefiriendo los canales culturales, de noticias, películas, deportes y fútbol. 
Afirman que en Chile la oferta cultural es escasa, y la que hay les parece cara. El país de comparación es Argentina, del cual destacan sus musicales. De una forma más positiva, desde el punto de vista de la oferta cultural, consideran que ha habido una masificación de los conciertos, a los cuales asisten con mucha frecuencia. Viajan dentro de Chile, y fuera, Argentina es un destino habitual.

siempre el que tiene plata es malo y la niñita buena es la pobre. Entonces también como que intentan siempre marcar una división. Es como un tema ahí en el cine y la televisión (Manuel).

mi papá vive en Argentina, y el argentino tiene la cultura del tiempo libre que acá no tenemos... (Manuel).

c) Mujeres C2. Afirman que su tiempo libre es dedicado principalmente a descansar. En este sentido, dicen leer el diario con tranquilidad durante el fin de semana. Salen a comer (cenar), van al cine, y es habitual que queden con sus amigos para tomar algo. A veces asisten a exposiciones de arte. Cuando eran niños o adolescentes, algunos recibieron algún curso de canto, guitarra o ajedrez.

... ahora compré las entradas para ir a los recitales que quiero este semestre, así como 3 meses antes. Voy a una exposición de vez en cuando, cuando puedo. Como vivo en el sector Lastarria, de repente salir a caminar, ver algo entretenido y pasar a mirar. Voy al cine con cierta frecuencia... (Juana).

d) Hombres C2. Su tiempo libre quieren pasarlo con sus seres queridos, principalmente con su familia. Lo dedican a descansar, leer e ir al cine. No obstante, sostienen que es un tiempo sobre todo dedicado a no hacer nada, a estar en blanco, a echarse en la tina (bañera) o en la cama. Algunos mencionan jugar al fútbol con los amigos un día a la semana. Leen el diario con calma el fin de semana ya que no lo pueden hacer por falta de tiempo durante los días de trabajo. Afirman que les gusta ir a los museos.

Quizás en otros países la gente entiende que su tiempo libre es donde activamente va y busca hacer cosas, actividades, va y busca... ¿qué sé yo? Eventos culturales, cine música, lo que sea, ir a pasear, pero aquí a mí me pasa que logro ordenar mis tiempos, que tengo poco, y lo ordeno para tener una, dos horas, casi como reloj, para poder tirarme a la cama y descansar (Antonio). 
...me gusta ir al museo y cuestiones así, pero de repente, ya llegar al centro es una joda, y estacionar, que cobran un ojo de la cara, después tenís que pagar la entrada... (Pedro).

e) Mujeres C3. Su tiempo libre es para estar con su familia, ver una película en la televisión o ir al cine. También lo asocian con comer algo rico, e incluso mencionan hacer yoga. Asimismo, hablan de las bondades de pasear, salir al aire libre y viajar. El mall no les gusta, pues dicen que les estresa. Les agrada despertarse más tarde, sin que suene el despertador. En la televisión, ven noticias y a veces algo de cultura. La televisión nacional las puede poner de mal humor, pues sus contenidos pueden ser considerados de mala calidad o «basura». Para informarse, varias dicen preferir el diario a la televisión. Ir al gimnasio les parece aburrido, salvo que el ejercicio se realice en compañía de alguna amiga. Sin embargo, sienten que es algo que debieran hacer, pues practicar algún deporte sería necesario.

...me encantaría, si tuviera más recursos, más tiempo, me encantaría viajar, conocer mi país de norte a sur (Anita).

f) Hombres C3. Dicen ir al cine. Disfrutan de comer algo rico. $\mathrm{Su}$ tiempo libre es asociado a descansar, tomar la siesta y no pensar en nada. Les gusta salir a caminar. Ven la televisión. Algunos se inclinan por ver una película antes que leer cuando están en casa. Si leen, afirman preferir la novela histórica.

g) Mujeres D. Manifiestan dedicar su escaso tiempo libre a descansar y ver la televisión. También a estar con su familia, aunque únicamente cuando esto es posible, pues todos deben realizar largas jornadas de trabajo. Si pueden salir de noche el fin de semana, suelen ir a un pub a tomar algo y conversar con las amigas.

h) Hombres D. El tiempo libre es para hacer deporte, dentro de lo cual mencionan el fútbol y el pool (billar). También es el momento de estar en casa con la familia y ver televisión. Asimismo, algunos afirman ir al persa (mercado popular) los sábados. Salen a pasear y a veces a comer con la familia, y sacan a los niños a andar en bicicleta. En todo caso, enfatizan que ellos sólo disponen de tiempo libre los fines de semana.

...para mejorar, tal vez no pueda estudiar nunca, pero tengo hijos y voy a tener nietos, por eso hay que mejorar la educación, porque la pobreza se combate con la educación y buenos estudios (Juan). 
TABLA 2: VARIABLES DE PARTICIPACIÓN CULTURAL ANALIZADAS

\begin{tabular}{|c|c|c|}
\hline $\begin{array}{l}\text { PARTICIPACIÓN EN } \\
\text { ORGANIZACIONES } \\
\text { (BINARIA) }\end{array}$ & $\begin{array}{l}\text { ACTIVIDADES } \\
\text { REALIZADAS } \\
\text { (FRECUENCIA) }\end{array}$ & $\begin{array}{l}\text { USO DE INTERNET } \\
\text { (FRECUENCIA) }\end{array}$ \\
\hline $\begin{array}{l}\text { Club deportivo (o grupo } \\
\text { de personas que se } \\
\text { organiza para practicar } \\
\text { un deporte) }\end{array}$ & $\begin{array}{l}\text { Escuchar radio o } \\
\text { música }\end{array}$ & Buscar información \\
\hline $\begin{array}{l}\text { Movimiento Guías y } \\
\text { Scouts }\end{array}$ & Ir al cine & Enviar y recibir e-mails \\
\hline $\begin{array}{l}\text { Agrupación o grupo de } \\
\text { hobbie o juego (juegos } \\
\text { de rol, } v . g . \text {., excluyendo } \\
\text { comunidades virtuales) }\end{array}$ & $\begin{array}{l}\text { Salir o conversar con } \\
\text { amistades }\end{array}$ & $\begin{array}{l}\text { Bajar y/o escuchar música } \\
\text { o videos }\end{array}$ \\
\hline $\begin{array}{l}\text { Organización o agrupa- } \\
\text { ción artística y/o cultural } \\
\text { (ejemplo: grupo de } \\
\text { teatro, grupo de música, } \\
\text { grupo de danza, etc.) }\end{array}$ & $\begin{array}{l}\text { Salir de compras o } \\
\text { vitrinear }\end{array}$ & Bajar programas \\
\hline $\begin{array}{l}\text { Grupo o tribu urbana } \\
\text { (hip hop, punk, poke- } \\
\text { mon, otaku, skinhead, } \\
\text { metalero, etcétera) }\end{array}$ & $\begin{array}{l}\text { Ver televisión } \\
\text { o videos }\end{array}$ & $\begin{array}{l}\text { Chatear (con amigos/as, } \\
\text { para buscar pareja, etcétera) }\end{array}$ \\
\hline Barra de fútbol & $\begin{array}{l}\text { Leer diarios, libros o } \\
\text { revistas }\end{array}$ & \begin{tabular}{|l|} 
Participar en comunidad \\
virtual o foros de discusión
\end{tabular} \\
\hline \multirow[t]{7}{*}{$\begin{array}{l}\text { Comunidad o grupo } \\
\text { virtual (grupo de chat, } \\
\text { foros, juegos, etcétera) }\end{array}$} & Hacer deportes & $\begin{array}{l}\text { Comprar, utilizar banca } \\
\text { electrónica o hacer trámites }\end{array}$ \\
\hline & $\begin{array}{l}\text { Navegar y/o jugar en } \\
\text { internet o computador }\end{array}$ & $\begin{array}{l}\text { Leer prensa y noti- } \\
\text { cias/revistas/textos }\end{array}$ \\
\hline & $\begin{array}{l}\text { Carretear, salir a fies- } \\
\text { tas o ir a bailar }\end{array}$ & Escuchar radio \\
\hline & $\begin{array}{l}\text { Disfrutar de la natura- } \\
\text { leza (salir al parque, al } \\
\text { campo, la playa o la } \\
\text { montaña) }\end{array}$ & Usar Fotolog o Blog \\
\hline & Estar con tu pareja & Usar Facebook \\
\hline & $\begin{array}{l}\text { Asistir o participar en } \\
\text { alguna organización }\end{array}$ & \\
\hline & $\begin{array}{l}\text { Realizar actividades } \\
\text { artísticas (baile, teatro, } \\
\text { pintura) }\end{array}$ & \\
\hline
\end{tabular}




\section{CONCLUSIONES}

La teoría del capital cultural subordinó a la juventud desde el momento en que la consideró un tiempo para la reproducción de clase. Y los estudios que han venido utilizando esta perspectiva, invisibilizaron su problemática al sostener que la edad era la variable teórica de referencia, de lo cual se deriva que sus diferentes momentos serían unidades susceptibles de ser descritas o analizadas. En este sentido, existirían los jóvenes, o los adultos, u otras categorías cualesquiera dentro del rango etario de la vida, y los análisis terminarían construyendo un perfil de las mismas. Esta es la deriva que hasta ahora han tenido algunos estudios realizados dentro del marco de la teoría mencionada, pero no es la única alternativa, ni la teoría en sí misma nos obliga necesariamente a ello.

En este estudio hemos mostrado como la teoría del capital cultural junto a la aproximación propia del estudio de campo pueden ayudar a explicar las importantes desigualdades que existen entre los jóvenes. No siendo suficiente recurrir a la ortogonalidad entre los capitales y la edad, es imprescindible analizar las características de la desigualdad presente entre aquellos. No obstante, habiéndolo hecho, hemos podido observar como el capital cultural y los recursos económicos están fuertemente asociados a las significativas diferencias que identificamos entre las personas encuestadas. Asimismo, hemos podido constatarlo a través del análisis de focus groups realizados con personas de diferentes grupos socioeconómicos. Sin embargo, a ello debemos agregarle un matiz relevante. Si efectivamente los capitales económico y cultural correlacionan fuertemente de forma positiva, el solapamiento no es total. Podríamos decir que si bien la acumulación de capital cultural está asociada a una mayor intensidad participativa de los jóvenes, los mayores grados de activismo cultural sólo se alcanzan cuando los recursos económicos son también elevados, muy probablemente por el costo que involucra un comportamiento de este tipo. En otras palabras, la cultura exige recursos de los que las personas disponen de manera desigual.

Pero sería limitado pensar que sólo importan los capitales. Incluso entre los jóvenes influyen las diferencias etarias. Esto significa que pequeñas diferencias de edad tienen un efecto significativo en su comportamiento. Así se puede observar como aquellos de menor edad (entre quince a diecinueve años), participan más en organizaciones culturales, muestran un mayor activismo cultural y una mayor frecuencia de uso de la tecnología de internet, si bien las diferencias en estas dos últimas dimensiones parecen menores que en la primera. 
Al igual que la edad, el género también importa, aunque su influencia es variable según temática. En este estudio hemos podido constatar su impacto preferente en el comportamiento participativo relativo a las organizaciones. Esto parecería influido por dos hechos. Por un lado, porque las mujeres podrían tender a asociarse menos que los hombres. Por otro, y sobre todo, porque los hombres dominan la participación organizativa en asociaciones deportivas, siendo las de fútbol de particular relevancia para ellos. Sea como fuere, hombres y mujeres jóvenes manifiestan tener perfiles de participación en organizaciones considerablemente diferentes, lo que no parece anunciar un mundo futuro en el que las diferencias de género sean una cuestión del pasado. Por el contrario, las distinciones de género seguirán estando presentes en el futuro cultural de la sociedad chilena. No obstante, la relevancia de esta diferencia parece menor en lo que respecta al activismo cultural y al uso de internet, aun cuando los hombres parecen tener también aquí una propensión un poco superior a la participación.

Toda esta evidencia nos lleva a concluir que no hay un joven, sino muchos jóvenes con capitales y recursos culturales diferenciados. No hay una juventud enfrentada a los adultos desde el punto de vista de la edad, sino una gradualidad etaria que también los atraviesa a ellos. No hay, finalmente, un momento de la vida en el que las reglas de la estructuración social son suspendidas o puestas entre paréntesis, sino que la juventud es informada por las mismas de manera radical. A este respecto, quizás es la visión idílica de la que los adultos son portadores la que a menudo convierte a los años adolescentes y de primera adultez en un paraíso al que el recuerdo ha borrado sus aristas más problemáticas. Pero nuestros sueños o recuerdos del ayer no son las realidades de hoy, y muy probablemente tampoco las de entonces. $\mathrm{Y}$ aunque nuestra memoria y el relato de la misma sean importantes, la sociología de los jóvenes no debe olvidar las condiciones que los producen como tales.

En definitiva, las desigualdades entre los jóvenes, aun utilizando una definición amplia de esta categoría, son patentes y están fuertemente influidas por la acumulación de los capitales económico y cultural. Por lo tanto, la juventud es un momento de la vida probablemente tan estructurado socioeconómicamente como cualquier otro. No cabe duda de que se trata de un período en el que las personas adoptan decisiones muy importantes, de las que posteriormente pueden o no sentirse responsables, sobre las cuales puede o no juzgárseles culpables. E igualmente no cabe duda de que esas decisiones se han adop- 
tado bajo regímenes de realidad social que desde el principio generaron condiciones radicalmente diferentes para unos y otros jóvenes.

SANTIAGO (CHILE), MARZO 2013

RECIBIDO: MARZO 2013

ACEPTADO: ABRIL 2013

\section{REFERENCIAS BIBLIOGRÁFICAS}

BAKER, SARAH y BRUCE COHEN (2008): «From Snuggling and Snogging to Sampling and Scratching: Girls' Nonparticipation in Community-Based Music Activities». Youth \& Society Vol. 39.

BENNETT, ANDY y KeITH KAHN-HARRIS (eds.) (2004): After Subculture. Critical Studies in Contemporary Youth Culture. Houndmills, Basingstoke: Palgrave Macmillan.

BennetT, Tony; Mike SaVage, Elizabeth Silva, Alan Warde, Modesto GAYO-CAL y DAVID Wright (2009): Culture, Class, Distinction. London: Routledge.

BERMÚDEZ, EMILIA (2001): «Consumo cultural y representación de identidades juveniles». Ponencia presentada en el Congreso LASA, celebrado en la ciudad de Washington D. C. del 6 al 8 de septiembre de 2001.

BERNASCONI, ORIANA y ESTEBAN PUENTES (2001): «Gasto en bienes y servicios culturales de los hogares del Gran Santiago 1988-1997». Informe del Ministerio de Planificación y Cooperación, Gobierno de Chile, julio.

BOLIN, GÖRAN (1999): «Producing cultures. The construction of forms and contents of contemporary youth cultures». Young Vol. 7.

BOURDIEU, PIERRE (2005): Las reglas del arte. Génesis y estructura del campo literario. Barcelona: Anagrama.

(1990): «La 'juventud' no es más que una palabra». Sociología y cultura. México: Grijalbo.

(1979): La distinction. Paris: Les Éditions de Minuit.

CATALÁn, CARLOS y Guillermo Sunkel (1990): «Consumo cultural en Chile: la élite, lo masivo y lo popular». Documento de Trabajo No455. Santiago: FLACSO.

— y PABLO TORCHE (2005): Miradas y perspectivas. Consumo cultural en Chile. Santiago: INE y CNCA.

CONSEJo NACIONAL DE LA CUltuRA Y LAS ARTES (2011): Segunda encuesta nacional de participación y consumo cultural. Valparaíso: CNCA. (2007): Encuesta de consumo cultural 2004-2005. Valparaíso: CNCA.

DE CASTRO, LuCÍA (2006): «What is new in the 'south'? Consumer culture and the vicissitudes of poor youth's identity construction in urban Brazil». Young Vol. 14(3). 
Modesto Gayo-Cal; Mike Savage y Alan Warde (2006): «A Cultural Map of the United Kingdom, 2003». Cultural Trends, 58/59, Vol. 15, issue $2 / 3$.

; BeRTA TeITElboim y MaRÍA LuISA MÉNDEZ (2009): «Patrones culturales de uso del tiempo libre en Chile. Una aproximación desde la teoría bourdieuana». Universum, Año 24, $\mathrm{N}^{\circ} 2$.

- ; María Luisa MÉNDEZ; Rosario RadaKovich y ANA WortMan (2011): «Consumo cultural y desigualdad de clase, género y edad: un estudio comparado en Argentina, Chile y Uruguay». Serie Avances de Investigación $\mathrm{N}^{\circ} 62$. Madrid: Fundación Carolina.

—-; Berta TeITELbOIM y MARÍA LuISA MÉNDEZ (2012): «Desigualdad cultural y estructura ocupacional. La pos-industrialización en Chile». Artículo inédito.

InSTITUTO NACIONAL DE LA JUVENTUd (2010): Sexta encuesta nacional de juventud. Santiago: INJUV.

HAGEN, INGUNN (2007): “'We can't just sit the whole day watching TV': Negotiations concerning media use among youngsters and their parents». Young Vol. 15.

JENSEN, SUNE Q. (2006): «Rethinking subcultural capital». Young Vol. 14(3).

KIILAKOSKI, TOMI y ATTE OKSANEN (2011): «Soundtrack of the School Shootings: Cultural Script, Music and Male Rage». Young Vol. 19.

LEE, LISA (2005): «Young people and the Internet: From theory to practice». Young Vol. 13.

LEONARD, MADELEINE (2008): «Social and Subcultural Capital Among Teenagers in Northern Ireland». Youth \& Society Vol. 40.

MugGLETON, DAVID (2005): «From Classlessness to Clubculture. A Genealogy of Post-war British Youth Cultural Analysis». Young Vol. 13(2).

SaVage, Mike; Modesto Gayo-Cal, Alan Warde, Gindo Tampubolon (con la colaboración de JOHS JJELLBREKKE, BRIGITTE LEROUX and HENRY ROUANET) (2005): «Cultural Capital in the UK: A Preliminary Report using orrespondence Analysis». RESC Working Paper Series $\mathrm{N}^{\circ} 4$.

$-\mathrm{y}-(2011)$ : «Unravelling the Omnivore: A Field Analysis of Contemporary Musical Taste in the United Kingdom». Poetics 39.

Selfhout, MaARTen; Marc Delsing, Ter Tom Bogt y Wim Meeus (2008): «Heavy Metal and Hip-Hop Style Preference and Externalizing Problem Behavior: A Two-Wave Longitudinal Study». Youth \& Society Vol. 39.

SHILDRICK, TRACY (2006): «Youth Culture, Subculture and the Importance of Neighbourhood». Young Vol. 14.

SUNKEL, GUILLERMO (2006) (coordinador): El consumo cultural en América Latina. Bogotá: Convenio Andrés Bello.

TERPSTRA, JAN (2006): «Youth Subculture and Social Exclusion». Young. Nordic Journal of Youth Research Vol. 14(2).

Tотн, CsabA (2008): «J-Pop and Performances of Young Female Identity: Music, Gender and Urban Space in Tokyo». Young Vol. 16. 
Vanden Abeele, MarieK y KeIth Roe (2011): «New Life, Old Friends: A Cross-cultural Comparison of the Use of Communication Technologies in the Social Life of College Freshmen». Young Vol. 19.

Wel, Frits W. VAN; WILLEMIJN MAARSINGH, TER TOM Bogt y QUINTEN RAAIJMAKERS (2008): «Youth Cultural Stypes. From Snob to Pop?». Young. Nordic Journal of Youth Research Vol. 16(3).

Wilska, Terhi-AnNa y SueIla Pedrozo (2007): «New Technology and Young People's Consumer Identitites: A Comparative Study between Finland and Brazil». Young, Nordic Journal of Youth Research Vol. 15(4).

WILLIS, PAUL (1977): Learning to labour. How working class kids get working class jobs. Aldershot: Gower. 